\title{
Abrupt appearance of the domain pattern and fatigue of thin ferroelectric films
}

\author{
A.M. Bratkovsky ${ }^{1}$ and A.P. Levanyuk ${ }^{2}$ \\ ${ }^{1}$ Hewlett-Packard Laboratories, 3500 Deer Creek Road, Palo Alto, \\ California 94304 \\ ${ }^{2}$ Departamento de Física de la Materia Condensada, CIII, Universidad \\ Autónoma de Madrid, 28049 Madrid, Spain
}

(August 11, 1999)

\begin{abstract}
We study the domain structure in ferroelectric thin films with a 'passive' layer (material with damaged ferroelectric properties) at the interface between the film and electrodes within a continuous medium approximation. An abrupt transition from a monodomain to a polydomain state has been found with the increase of the 'passive' layer thickness $d$. The domain width changes very quickly at the transition (exponentially with $d^{-2}$ ). We have estimated the dielectric response $d P / d E$ (the slope of the hysteresis loop) in the 'fatigued' multidomain state and found that it is in agreement with experiment, assuming realistic parameters of the layer. We derive a simple universal relation for the dielectric response, which scales as $1 / d$, involving only the properties of the passive layer. This relation qualitatively reproduces the evolution of the hysteresis loop in fatigued samples and it could be tested with controlled experiments. It is expected that the coercive field should increase with decreasing lateral size of the film. We believe that specific properties of the domain structure under bias voltage in ferroelectrics with a passive layer can resolve the long-standing "paradox of the coercive field'.
\end{abstract}

77.80.-e, 77.80.Dj, 84.32.Tt, 85.50.+k

Recent studies of thin ferroelectric films have revived interest in properties of the ferroelectric domain structures. It became clear that some basic aspects of these properties remain unexplored, whereas they are of key importance for applications. For instance, the progressive loss of switchable polarization after repeated switching cycles, i.e. polarization fatigue, is a serious problem in device applications of ferroelectrics. In spite of extensive studies, the physics of the fatigue remains poorly understood. Various mechanisms were considered over the years [1 1 ]. In many cases the deterioration of the switching behavior, like the loss of the coercive force and of the squareness of hysteresis loop, were attributed to the growth of a 'passive layer' at the ferroelectric-electrode interface [1] 3. 6], or to the pinning of domain walls by defects [2,4]. In this paper, we study the effects of a passive layer (material with damaged ferroelectric properties). We assume an ideal infinite ferroelectric film and treat it within the continuous medium approximation. The model exhibits very interesting properties relevant for real systems.

The passive layer leads to the appearance of a depolarizing field in the ferroelectric, so that the system would tend to transform to a polydomain state in order to reduce the field. There is, therefore, a transition between the monodomain state, when the thickness $d$ of the passive layer is zero, to a polydomain state otherwise. What is the nature of this transition? This is the main theoretical question we address in this paper.

For a short-circuited infinite ferroelectric plate, as we shall show, the domain structure exists for any value of the passive layer thickness, in disagreement with some earlier results [9]. A surprising feature of the present study is that the formation of the polydomain state sets in abruptly with the appearance of the passive layer. The period of the domain structure tends to infinity exponentially when the passive layer thickness $d$ goes to zero. The theory allows one to study the evolution of the domain structure in the asymptotic regime of very wide domains and calculate its dielectric response.

The slope of the resulting $P-E$ (polarization - electric field) hysteresis curve at $E=0$ is directly related to the thickness $d$ and dielectric constant $\epsilon_{g}$ of the nonferroelectric layer. We show that there is a universal relation (14) $d P / d E \propto \epsilon_{g} / d$. This relation is in good correspondence with available experimental data. It is important that even without pinning by defects, the response of this structure to an external bias voltage is rigid. The implication for real systems is that with the growth of the passive layer the hysteresis loop very quickly deteriorates and looses its squareness.

The properties of the domain structure in the presence of a passive layer are important for the problem of switching in ferroelectric thin films. If we were to apply the external field in the opposite direction to the spontaneous polarization in a monodomain state, the equilibrium size of the domain with the 'wrong' polarization would eventually become less than the film size. Then a new domain wall would appear in the film and we get a switching. Obviously, the coercive field for this mecha- 
nism should then increase with decreasing lateral size of the film.

We shall consider a ferroelectric film under a bias voltage $U$ with thickness $l$ separated from the top and bottom electrodes by passive layers with thickness $d / 2$ (Fig. 1, inset). For a system with a given voltage on the electrodes, the equilibrium distribution of charges and polarization corresponds to the extremum of the thermodynamic potential $\tilde{F}$, which includes explicitly the work produced by the voltage source(s) and the energy of the electric field 10, 14, 97:

$$
\tilde{F}=F_{\mathrm{LGD}}+\int d V E^{2} /(8 \pi)-\sum_{a}^{\text {electrodes }} e_{a} \varphi_{a}
$$

Here $F_{\text {LGD }}$ is the standard Landau-Ginzburg-Devonshire (LGD) functional at zero electric field [10,12], $\vec{E}$ is the electric field, while $e_{a}\left(\varphi_{a}\right)$ is the charge (potential) on the electrode $a$. The last term in Eq. (11) accounts for the work of the external voltage source(s).

We are interested in a case when the ferroelectric has a spontaneous polarization $\vec{P}_{s} \| z$ (Fig. 1, inset). The film has a dielectric constant $\epsilon_{c}\left(\epsilon_{a}\right)$ in the $z$-direction (in the $x y$-plane), and the dielectric constant of the passive layer is $\epsilon_{g}$. We select the $x$-axis perpendicular to the domain walls. The potential $\varphi(\vec{E}=-\nabla \varphi)$ satisfies the following equations in the ferroelectric and the passive layer,

$$
\epsilon_{a} \frac{\partial^{2} \varphi_{f}}{\partial x^{2}}+\epsilon_{c} \frac{\partial^{2} \varphi_{f}}{\partial z^{2}}=0, \quad \frac{\partial^{2} \varphi_{g}}{\partial x^{2}}+\frac{\partial^{2} \varphi_{g}}{\partial z^{2}}=0
$$

with the boundary conditions $\varphi=-(+) U / 2, \quad z=$ $+(-)(l+d) / 2$, and

$$
\epsilon_{c} \frac{\partial \varphi_{f}}{\partial z}-\epsilon_{g} \frac{\partial \varphi_{g}}{\partial z}=4 \pi \sigma(x), \quad \varphi_{f}=\varphi_{g}, \text { at } z=l / 2,
$$

where the subscript $f(g)$ denotes the ferroelectric (passive layer, or a vacuum gap). Here $\sigma$ is the density of the bound charge due to spontaneous polarization at the ferroelectric-passive layer interface, $\sigma=P_{n s}= \pm P_{s}$, depending on the normal direction of the polarization at the interface, alternating from domain to domain, Fig. 1(inset). We have assumed a usual separation of linear and spontaneous polarization, so that the displacement vector is $D_{i}=\epsilon_{i k} E_{k}+P_{s i}$, where $i, k=x, y, z$, and the dielectric response $\epsilon_{i k}$ in uniaxial, Fig. 1(inset).

We shall assume that the film is split into domains with alternating $z$-component of polarization and widths $a_{1}$ and $a_{2}$ so that the pattern is periodic with a period $T=a_{1}+a_{2}$. This assumption is perfectly reasonable, since the bound charges at the boundary between the ferroelectric and the passive layer are fully compensated by the image charges at the electrodes. In this situation the domains would not branch (see e.g. [12], p.219). The solution of the Poisson equations (2) is then readily found by Fourier transformation

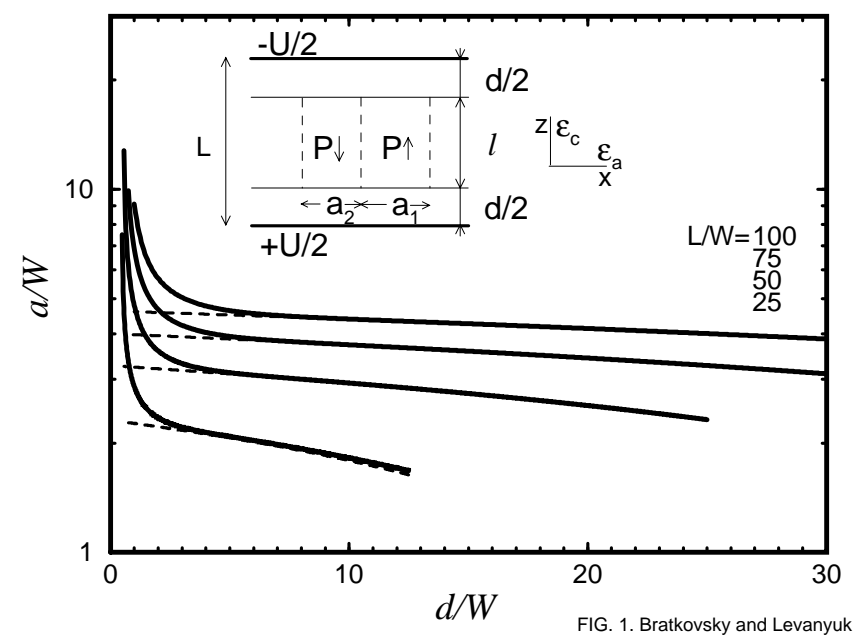

FIG. 1. The domain width $a$ in a ferroelectric capacitor versus the passive layer thickness $d$ for different separations between the electrode plates $L, \epsilon_{g}=\epsilon_{a}=10, \epsilon_{c}=200$ at zero bias. Dashed lines show the behavior of the Kittel width $a_{K}$. All quantities are scaled to $W$, the domain wall thickness [12]. Inset shows schematics of the electroded ferroelectric film (capacitor) under bias voltage $U$. Note that the transition to very wide domains at small $d$ is very sharp, even when shown on a logarithmic scale.

$$
\begin{aligned}
\sigma(x) & =\sum_{n=-\infty}^{\infty} \sigma_{n} e^{\frac{2 \pi i n x}{T}}, \\
\sigma_{n} & =\frac{P_{s}}{i \pi n}\left[1-\exp \left(\frac{2 \pi i n a_{1}}{T}\right)\right], \quad n \neq 0, \\
\sigma_{n=0} & \equiv \sigma_{0}=P_{s} \frac{a_{1}-a_{2}}{a_{1}+a_{2}} \equiv P_{s} \delta, \\
\varphi_{\alpha}(x, z) & =\sum_{n=-\infty}^{\infty} \varphi_{n \alpha}(z) e^{\frac{2 \pi i n x}{T}},
\end{aligned}
$$

where $n$ is the number of the Fourier component, and $\alpha=f, g$. With the assumption that $P_{s}=$ const inside the domains, the free energy Eq. (1) becomes $\tilde{F}=F_{0}+$ $\frac{1}{2} \int_{\mathrm{FE}} d \mathcal{A} \sigma \varphi-\frac{1}{2} \sum_{a} e_{a} \varphi_{a}$, where $\bar{F}_{0}=F_{\mathrm{LGD}}\left[P_{s}\right]$ is the free energy of the ferroelectric with the polarization $P_{s}$ which contains the surface energy of the domain walls, and the integral is taken over the interface between the ferroelectric and the passive layer. It is useful to treat the homogeneous part $(n=0)$ of the potential and the surface charge separately from other terms. Then the result for the (dimensionless) free energy per unit volume is

$$
\begin{aligned}
f \equiv & \frac{\tilde{F}}{\mathcal{A} L P_{s}^{2}}=\frac{\delta}{2} \frac{(4 \pi \delta d / L)-\epsilon_{g} u}{\left(\epsilon_{c} d / l\right)+\epsilon_{g}} \\
& -\frac{\epsilon_{g} u}{8 \pi} \frac{\left(\epsilon_{c} u L / l\right)+4 \pi \delta}{\left(\epsilon_{c} d / l\right)+\epsilon_{g}}+f_{K}(t)+f_{x}(t, \delta, u),
\end{aligned}
$$

where the dimensionless bias voltage is $u \equiv U / P_{s} L$, and the first two 'homogeneous' terms describe the growth 
of the domains with the 'right' polarization (along the external field) at the expense of domains with opposite polarization, $f_{K}(t)$ is the Kittel-like free energy [13, 11 defining the dimensionless period of the domain structure $t \equiv\left(a_{1}+a_{2}\right) / L$, and $f_{x}(t, \delta, u)$ is the cross-term 14] describing, in addition to the first two terms, the appearance of a net polarization in the film under bias, while $L=l+d$ is the distance between the electrodes, Fig. 1(inset). To find the equilibrium distribution of charges and polarization, this functional should be varied with respect to $\delta$ and $t$ at constant bias voltage (parameter $u$ ).

The domain structure with the passive layer can be determined simply from a minimum of $f_{K}$ in the case of zero bias voltage, when $a_{1}=a_{2}=a$, and

$$
\begin{aligned}
& L f_{K}=\frac{\Delta l}{a}+\frac{32 a}{\pi^{2}} \sum_{j=0}^{\infty} \frac{1}{(2 j+1)^{3}} \\
& \times \frac{1}{\epsilon_{g} \operatorname{coth}\left[\frac{(2 j+1) \pi d}{2 a}\right]+\sqrt{\epsilon_{a} \epsilon_{c}} \operatorname{coth}\left[\left(\frac{\epsilon_{a}}{\epsilon_{c}}\right)^{1 / 2} \frac{(2 j+1) \pi l}{2 a}\right]} .
\end{aligned}
$$

For a free standing film, $d \gg l$, Eq. (9) has a standard (generalized Kittel) solution

$$
\begin{aligned}
a & =a_{K} \equiv\left[\pi^{2}\left(\epsilon_{g}+\sqrt{\epsilon_{a} \epsilon_{c}}\right) \Delta l / 28 \zeta(3)\right]^{1 / 2} \\
& =\left[0.3\left(\epsilon_{g}+\sqrt{\epsilon_{a} \epsilon_{c}}\right) \Delta l\right]^{1 / 2},
\end{aligned}
$$

where $\Delta$ is the (temperature dependent) characteristic length, which is proportional to the product of the inverse of the dielectric susceptibility $\chi$ in the FE phase and the domain wall thickness $W$ [11,12].

Most interesting is the ferroelectric film with a narrow passive layer, $d \ll l$. When $\left(\frac{\epsilon_{a}}{\epsilon_{c}}\right)^{1 / 2} \frac{\pi l}{2 a} \gtrsim 1$, the second coth in (9) can be replaced by unity and neglected altogether in all terms in the sum with $j \lesssim a /(\pi d)$, since the first factor in the denominator is $\epsilon_{g} \operatorname{coth}\left[\frac{\pi d}{2 a}(2 j+1)\right] \sim$ $\frac{2 a}{\pi d} \gg 1$. We see that the factor a cancels out from all those terms in the sum. The dependence of the second term (the so-called energy of emergence per unit area) on $a$ becomes very weak, and the free energy minimum would correspond to very large domain widths, $a \gg a_{K}$, Fig. 1. The domain size may exceed the lateral size of the sample, and then we would have a monodomain film. This surprising behavior is the result of the effective screening of the bound charges on the ferroelectricpassive layer interface by the metallic electrodes [15].

To analyze the transformation between monodomain and multidomain states, we first consider an exactly solvable model with $\epsilon_{g}=\sqrt{\epsilon_{a} \epsilon_{c}}$ in the regime $l / a \gtrsim 1$. Then

$$
\begin{aligned}
L f_{K} & =\Delta l / a+\frac{32 a}{\pi^{2} \epsilon_{g}} \sum_{j=0}^{\infty} \frac{1}{(2 j+1)^{3}} \frac{1}{\operatorname{coth}\left[\frac{(2 j+1) \pi d}{2 a}\right]+1} \\
& =\Delta l / a+\frac{16 d}{\pi \epsilon_{g}} I, \quad \text { where }
\end{aligned}
$$

$$
I \equiv \int_{0}^{1} \mathrm{~d} \lambda \sum_{j=0}^{\infty} \frac{e^{-\lambda(2 j+1) \pi d / a}}{(2 j+1)^{2}}=\frac{\pi^{2}}{8}-\frac{\xi}{4} \ln \frac{e^{p}}{\xi},
$$

up to terms $\sim \xi^{3}$, where $\xi \equiv \pi d / a \ll 1$, and $p=(3+$ $\ln 4) / 2$ [16]. Minimizing the free energy $f_{K}$, we finally obtain the width of the domains in the case of a narrow passive layer

$$
a=\frac{\pi d}{2 e^{1 / 2}} \exp \left(\frac{\epsilon_{g} \Delta l}{4 d^{2}}\right)=0.95 d \exp \left(0.4 \frac{a_{K}^{2}}{d^{2}}\right)
$$

This unusual solution is extremely sensitive to the thickness $d$ of the passive layer, so that the domain width increases explosively for narrowing passive layer. This conclusion is general, it applies at $a \gtrsim l$ too, since the cancelation of $a$ persists in this case as well, as illustrated by the results of the exact calculation in Fig. 1. Note that a similar case of a ferroelectric capacitor with nonferroelectric insulating layer at the electrode surface has been considered recently [17, but the exponential growth of domain thickness (12) was overlooked, and only the standard Kittel expression was obtained.

We have seen that the ferroelectric film splits into domains as a result of the growth of a passive layer at the electrode surfaces. This substantially decreases the coercive field. It will be defined by only the strength of the domain wall pinning. We can assess the validity of this scenario of fatigued state by considering an ideal case with no pinning and comparing the estimated slope of the hysteresis loop with experiment. To this end, we shall estimate the value of $d P_{a} / d E$, where $P_{a}$ is the apparent (measured) polarization (surface charge) $P_{a}=\sigma_{0}=P_{s} \delta$, Eq. (6), and $E$ is the electric field (in fact, it is more convenient to find $\left.d P_{a} / d U=L^{-1} d P_{a} / d E\right)$. The linear response to bias voltage is obtained from Eq. (8), which, for small bias $U$, takes the form

$$
f=f_{0}(t)+\frac{1}{2} S \delta^{2}-R u \delta,
$$

where $S$ is the domain structure stiffness with respect to changes in apparent polarization $P_{a}$, and the coefficient $R$ defines the response to the bias voltage. The slope of the hysteresis loop $L\left(d P_{a} / d U\right)=R / S$ is shown in Fig. 2. Since the cross-term $f_{x}$ in Eq. (8) changes only the numbers, we can obtain a simple analytical approximation by neglecting this term to the first approximation. The result is

$$
\frac{d P_{a}}{d U}=\frac{\epsilon_{g}}{4 \pi d} .
$$

This remarkably simple relation is universal, and depends only on properties of the passive layer the expression reads $\epsilon_{0} \epsilon_{g} / d$ in SI units]. It is also rather accurate in comparison with the exact numerical calculation, Fig. 2. It is well known from experiment that, as fatigue progresses ( $d$ grows), the slope of the hysteresis 


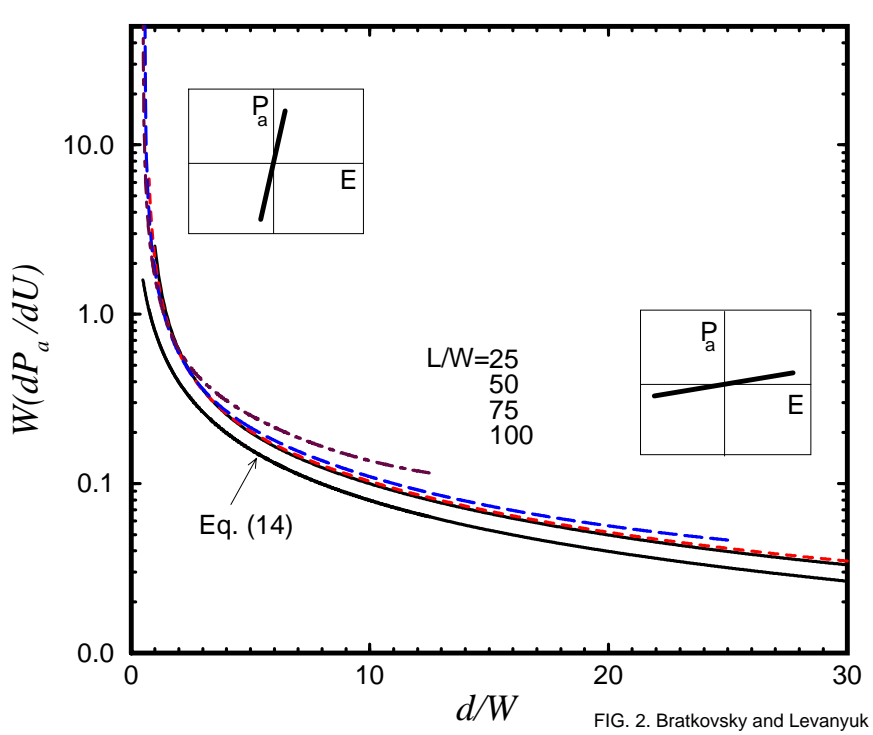

FIG. 2. The calculated slope of the hysteresis loop, $W\left(d P_{a} / d U\right)$, in a fatigued sample in comparison with the approximate expression. Eq. (14). The parameters of the film are the same as in Fig. 1. Insets show schematics of the very fast decrease of the slope of the hysteresis $P_{a}-E$ loop with the growth of the passive layer (fatigue).

loop steadily decreases 3, 3, 18. 18 . This behavior follows immediately from our Eq. (14), the slope of hysteresis loop decreases very rapidly with increasing $d$, Fig. 2 . From experiment [18], we can determine the slope in PZT films as $d P_{a} / d U \sim 15 \mu \mathrm{Ccm}^{-2} \mathrm{~V}^{-1}$. One can estimate the corresponding thickness of the passive layer from Fig. 2. Equation (14) gives for the passive layer thickness $d \sim 2-7 \mathrm{~nm}$ with $\epsilon_{q}=3-10$, the thickness of the film was $L=256 \mathrm{~nm}[18]$. This $d$ may be somewhat underestimated since (i) the experimental slope is affected by pinning of the domain wall, which we neglected, and (ii) Eq. (14) somewhat underestimates the exact result (Fig. 2). The result shows that already a tiny passive layer has a profound effect on the hysteresis loop and its slope rapidly diminishes with the growth of the passive layer. Thus, in thin films with small lateral dimensions the transition into a fatigued state with domains will be discontinuous and the growth of the passive layer can easily trigger this transformation.

Within our model there is no hysteresis for an infinite film and the dielectric response describes the slope of the hysteresis loop. It is important to emphasize that the film becomes more 'dielectrically rigid' because of the growth of the passive layer alone, even without taking into account either bulk or surface pinning of the domain walls. The estimated slope of the fatigued system is in good correspondence with experimental data, so that the growth of the passive layer might be the main origin of fatigue.

We believe also that the specific features of properties of the domain structure in the presence of the passive layer may be a key to resolving the famous 'paradox' of the coercive field. All the estimates for creating a nucleus with reversed polarization give extremely large values of the energy barrier, $E^{*} \sim 10^{7} k_{B} T[1921]$. Such a huge barrier makes a thermal nucleation impossible, and this makes the related estimates of the coercive field questionable 19 21.

In our scenario, however, the coercive field is defined by the energy barrier for the domain wall to 'enter' the sample. It is likely to be much smaller than the classic barrier for nucleation $E^{*}$, and this possibly resolves this long-standing paradox. We hope that these new ideas about the problem of switching will prove fruitful. For instance, we expect that in thin films the coercive field will be increasing with decreasing lateral size of the sample, since the size of a domain with a 'wrong' polarization should become smaller than that size. This should be contrasted with the usual conclusion that the coercive field grows with decreasing thickness of the sample [22], irrespective of its lateral size. It would be very interesting to test this prediction experimentally, especially in view of ever smaller sizes of films used in devices.

The effect of the passive layer on switching in the ferroelectric films is a specific case of a more general problem: the effect of inhomogeneities in the system on its ferroelectric properties. As we have shown elsewhere [23], a similar mechanism produces the fatigue of a film without any actual passive layer, in presence of a depletion charge in ferroelectric-semiconducting films 24 .

It is worth emphasizing that none of our conclusions depends on a particular form for the domain structure (which may be of a checkerboard type, etc.) This is because the effect of partial screening of the depolarizing field by electrodes obviously applies to any domain pattern.

We appreciate useful discussions with J. Amano, G.A.D. Briggs, A. Gruverman, and R.S. Williams. Extensive conversations with L. Wills-Mirkarimi were invaluable. AL would like to acknowledge a support and hospitality of Quantum Structures Research Initiative at Hewlett-Packard Labs (Palo Alto) during the course of this work.

[1] M.E. Drougard and R. Landauer, J. Appl. Phys. 30, 1663 (1959).

[2] D.M. Smyth, Curr. Opinion in Sol. St. Mater. Sci. 1, 692 (1996).

[3] S.L. Miller et al. J. Appl. Phys. 68, 6463 (1990).

[4] D.J. Taylor et al. Integr. Ferroelectrics 7, 123 (1995). 
[5] H.M. Duiker et al. J. Appl. Phys. 68, 5783 (1990); S.B. Desu and I.K. Yoo, Integr. Ferroelectrics 3, 5783 (1993).

[6] V.V. Lemanov and V.K. Yarmarkin, Phys. Sol. State 38, 1363 (1996) [Fiz. Tverd. Tela 38, 2482 (1996)].

[7] A.K. Kholkin et al. Appl. Phys. Lett. 68, 2577 (1996).

[8] A. Gruverman, O. Auciello, and H. Tokumoto, Appl. Phys. Lett. 69, 3191 (1996).

[9] E.V. Chensky and V.V. Tarasenko, JETP 56, 618 (1982) [Zh. Eksp. Teor. Fiz. 83, 1089 (1982)].

[10] L.D. Landau and E.M. Lifshitz,Electrodynamics of Continuous Media (Elsevier, 1985), (a) Secs. 5, 10, 19.

[11] Ref. 10], Sec. 44.

[12] B.A. Strukov and A.P. Levanyuk, Ferroelectric Phenomena in Crystals (Springer, Berlin, 1998).

[13] C. Kittel, Phys. Rev. 70, 965 (1946); T. Mitsui and J. Furuichi, Phys. Rev. 90, 193 (1953).

[14] A.M. Bratkovsky and A.P. Levanyuk (to be published).

[15] Such a screening is absent in magnetic systems, hence the behavior considered here has completely different origin compared to magnetic domains [cf. A. Hubert and R. Schäfer, Magnetic Domains (Springer, Berlin, 1998)].

[16] $I$ has been transformed similar to 11, 13 and $\sum_{n=1}^{\infty} b^{n} / n^{2}=\pi^{2} / 6-\int_{1}^{b}[\ln (1-x) / x] \mathrm{d} x$ was used to perform the series expansion.

[17] Y. Watanabe, Phys. Rev. B 57, 789 (1998); J. Appl. Phys. 83, 2179 (1998).

[18] A.K. Tagantsev et al. J. Appl. Phys. 78, 2623 (1995).

[19] R. Landauer, J. Appl. Phys. 28, 227 (1957).

[20] V. Janovec, Czech. J. Phys. 8, 3 (1958).

[21] H.F. Kay and J.W. Dunn, Phil. Mag. 7, 2027 (1962).

[22] A.K. Tagantsev et al., Integr. Ferroelectrics 6, 309 (1995); Integr. Ferroelectrics 4, 1 (1994).

[23] A.M. Bratkovsky and A.P. Levanyuk, Phys. Rev. B 61, Apr 1 (2000); cond-mat/ 9908070.

[24] P.W. Blom et al. Phys. Rev. Lett. 73, 2107 (1994). 